\title{
Live P2P Streaming System with High Playback Continuity
}

\author{
Meng Xianfu, Wu Wei \\ School of Computer Science and Technology, Dalian University of Technology, Dalian 116024, China \\ xfmeng@dlut.edu.cn
}

\begin{abstract}
P2P streaming system effectively fixed the overload and weak scalability shortcomings of C/S structures. It's working principle is divided into tree multicast and mesh multicast. Compared to tree multicast method, mesh multicast method based on Gossip protocol has the characteristics of high fault tolerance and high bandwidth utilization. Gossip multicast in P2P streaming system has become the mainstream. Data transfer of gossip protocols in high-dynamic environment is uncertainty, and the problems of slow start and poor playback smooth, easily happened in streaming media system, will seriously affect the user experience. This paper designs a real-time streaming system DHTPatch, based on the idea of "the capable ones are the busiest". We organize the nodes with better performance into a DHT network, DHT network can freely and accurately access to resources, make up for the disadvantage of gossip multicast data transmission. The experiment results show that the system not only improves the gossip streaming playback continuity, but also speeds up the streaming startup of the playback.

Index Terms - stream media, gossip, DHT, multicast, P2P
\end{abstract}

\section{Introduction}

In recent years, the multimedia service and application develop rapidly in Internet. According to the forecast of Cisco, the traffic of streaming system will constitute the $90 \%$ of Internet traffic till 2013 [1]. Most of commercial streaming systems, such as Youtube hulu, all based on CDN system, and the CDN system is helpful to reduce the overload and latency of streaming system, so it can serve more clients. However, the shortcoming of CDN is the expensive deployment cost. If we want to supply high quality video services, the performance and bandwidth of CDN server must be improved as the growth of users. P2P networks become a choice to replace the CDN system, since in P2P network, users not only download the video data, but also upload data to other users, and thus the utilization of upload bandwidth makes the P2P streaming system economical compared to CDN system.

Live streaming media services such as Internet TV disseminate video data to a large number of users. In these applications, multicast has become the most effective way for data dissemination. Since IP multicast requires hardware support, it's hard to spread in the actual network. Therefore, the researchers gradually shifted their attention to application layer multicast (ALM).

Recently, Gossip based ALM has become the mainstream in P2P streaming media systems. The basic Gossip protocol algorithm maintains a certain number of neighbors' information in each node, a node sends or receives a data block which randomly spread to parts of the neighbor nodes. The randomness of data dissemination and the flexibility of neighbor maintenance keep Gossip P2P streaming media system from significant performance degradation because of node failure, thus the protocol is well adapted to high dynamic network environment. The Gossip protocol running on the unstructured $\mathrm{P} 2 \mathrm{P}$ network, with high scalability and natural robustness, is very suitable for large-scale live media streaming systems. Yet, the uncertainty of data transmission as the shadow follows, caused by the dynamic nature of the network. Therefore, it is difficult to guarantee that each data block can reach every node in the network, which caused the video stream playback unsmooth [2] and slow start [3], seriously harming the user experience. As mentioned above, how to improve playback continuity and the speed of startup should be the key problem in streaming media system research [4-6].

Based on structured DHT network with fast and accurate data positioning in the global network, this paper designs a new overlay network structure DHTPatch, which could play a better support for live stream media system, and designs the corresponding data scheduling algorithm. Our main work is following:

A. Considering the node heterogeneity in streaming media network, based on the idea of "an able man is always busy", the system burdens the high performance nodes more works, improving the utilization of network source.

B. Using the ability of fast and accurate data acquisition in DHT network to make up for the uncertainty data transmission of gossip multicast, the system effectively improves the availability of video data and the playback continuity.

C. We use the consistent hashing function of DHT network to distribute resources evenly, improve the slow data diffusion of pull-data gossip protocol, and increase the mean startup speed of video playback.

\section{The Structure of DHT Patch}

The heterogeneity of nodes is an important feature of P2P network. Users differ from each other from bandwidth, process ability, storage, NAT access and so on. The node in DHTPatch has two statuses: normal node, executing gossip multicast protocol data pre-fetching operation. DHT node, except for the operation of gossip multicast, the node affords the operation to maintain the DHT structure and data prefetching, and the DHT buffer in DHT node stores the prestored video data from server. As Fig.1 shows, the dashed box module is unique to DHT node. If the data in DHT buffer can be used in Play buffer, the node can use it directly instead of 
searching in the network, and the DHT buffer needs to eliminate the expired data in FIFO mode.

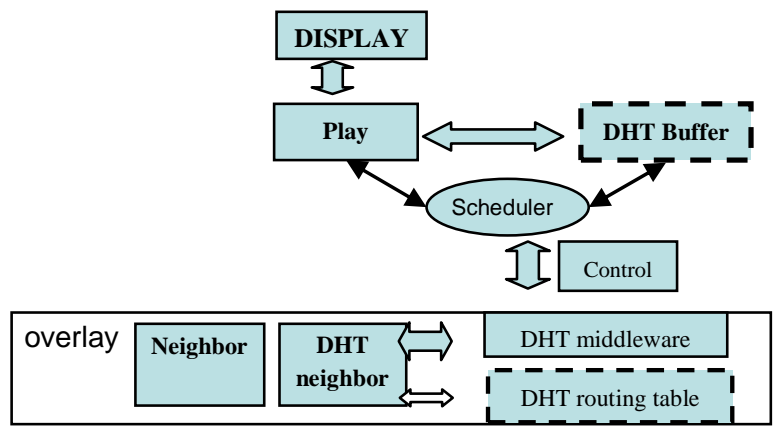

Fig. 1 DHTPatch system design

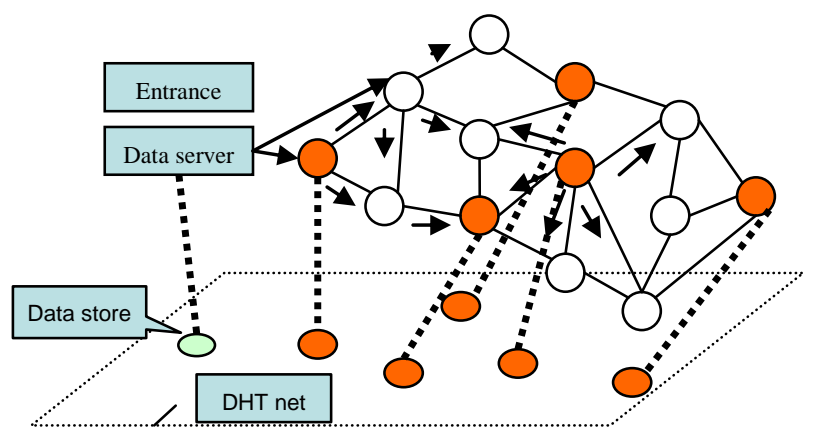

Fig. 2 DHTPatch topology

Fig 2 shows the structure of the DHTPatch. The data server disseminates streaming media data to the network, and the data server is a fixed node in the DHT subnet, and distributes data blocks to DHT network. The DHT network can be any existing DHT network such as Chord, Tapestry, kademlia, etc. These network structures have the ability to accurately position data, and since they use consistent hashing function so that they are good in load balancing and suitable for large-scale distributed data systems.

The entrance server assists nodes in joining network, as a public entrance, the server is also responsible for the selection of nodes for the DHT network. It will be discussed in detail in the following. High performance nodes constitute a DHT network, which store the data from data server in DHT mode; DHT nodes except data server only provide data passively, avoiding the redundancy caused by inserting data into DHT network. In the upper layer of the unstructured network, it still runs the normal gossip multicast to disseminate video data.

\section{A. Node joining}

A newly added node gets a list containing a small number of nodes (peer table) from the entrance server. The list consists of normal nodes and DHT nodes, and the new node also can acquire more nodes from neighbors as candidates for neighbors, and adds them into peer table. If it fails to obtain the node from the entrance server, the the node notifies the entrance server to update the stored node list. The node selects the neighbor that have the least latency.

The DHTPatch system needs to choose the node with high performance to constitute DHT network, so as to make full use of node capacity. In streaming media system, all the nodes join the network through a public entrance server, which provides a unified measurement for the node performance evaluation. We examine the performance of a node by three properties: (1) Processing ability of the CPU (CPU); (2) Memory size (Memory); (3) Bandwidth. The node performance can be represented by $\mathrm{NP}=\mathrm{W} 1 * \mathrm{CPU}$ $+\mathrm{W} 2 *$ Memory $+\mathrm{W} 3 *$ Bandwidth (W1, W2 and $\mathrm{W} 3$ are the weights).

The initial DHT network only contains one node - the data server. The entrance server calculates the performance value NP of each node. The process of building DHT subnet is divided into two stages: (1) the starting stage: the server keeps a list of node information and their NP value, the threshold value is set to $N P_{D H T}=$ $\operatorname{MIN}\left(N P_{\text {node }}\right)+\alpha\left(\operatorname{MAX}\left(N P_{\text {node }}\right)-\operatorname{MIN}\left(N P_{\text {node }}\right)\right)$. If the node has a greater NP than the threshold, the DHT joining algorithm is executed, otherwise, it's still an ordinary node.

\section{B. Threshold adjustment}

With the nodes joining, the threshold needs to be constantly adjusted. If node $\mathrm{i}$ has greater NP than $N P_{D H T}$ then we have it join the DHT network, and also the entrance server calculates the new value of $N P_{D H T}=\beta^{*} N P_{i}+(1-\beta) N P_{D H T}$. Thus the new nodes continuously join the DHT network, and a certain proportion of nodes access the DHT network. It remains the number of DHT node at a certain level.

\section{Node leaving and neighbor table maintenance}

Because the DHT subnet is based on the existing DHT network, the topology structure maintenance needs not discuss. In the unstructured network of upper layer, nodes exchange information periodically, and thus the node failure can easily be found, and the node can easily modify the neighbor table. Data maintenance is divided into two kinds: the node leaving with notification. According to the DHT network protocol, such as Chord network, in such case the data will be transferred to the successor node; node failure. Because the timeliness of live video streaming media data, missing data will gradually become invalid, and data redundancy in data scheduling also can prevent video system from data loss.

Since the normal nodes are not in the DHT network, if a normal node want to get video data through the DHT network rapidly and accurately, we need a middle-ware to provide the service for shielding the difference of ordinary nodes and DHT nodes. A normal node can locate the required data in the $\log \mathrm{N}$ time. Thus normal node and DHT node can quickly access to the required data through DHT network.

\section{Data Scheduling}

In DHT Patch system, each node and its connected neighbors periodically exchange their cached data 
information. We call an exchange cycle the scheduling period. In each scheduling period, data dispatcher first identifies the connection neighbors whose data blocks are available in the cache, and then checks whether there exists better choice in DHT subnet, and then pulls data from the provider.

The effect of data scheduling depends on the transmission capacity of the data providers and the problem of expired data in live streaming media. DHT-Patch has a special structure, and a node gets video data from the two places: (1)stream data spread from the gossip protocol; (2)the data blocks allocated through DHT network. Data in Play Buffer can be obtained from the DHT buffer and diffuse through DHT network. The data from the latter reach the desired node fast than the former, part of the data can "in advance" reach the node, reducing download time of data for playback.

Data blocks are measured by two aspects: the rarity and the urgency. The rarity of data block $D_{i}$ is defined as rarity $_{i}=1 / n_{i}$, where $n_{i}$ represents the number of neighbors the $D_{i}$ has; urgency is defines as urgent $_{i}=1 /$ deadline . Let C be the size of data blocks, $R_{i}$ is the data transmission rate, $\mathrm{PR}$ is the playback speed, the deadline is the latest time before playback, deadline $=\left(I D_{i}-I D_{\text {play }}\right) / P R-C / R_{i}$

The priority of each block is expressed as:

priority $_{i}=\max \left(\frac{\text { urgent }_{i}-\min (\text { urgent })}{\max (\text { urgent })-\min (\text { urgent })}, \frac{\text { rarity }_{i}-\min (\text { rarity })}{\max (\text { rarity })-\min (\text { rarity })}\right)$

DHT subnet stores redundancy data to prevent the data blocks from failing to be obtained caused by node failure, for a data block $n$, we calculate

$$
\operatorname{hash}(n \times i) \% N(i=1,2, \ldots, k)
$$

to get $\mathrm{k}$ IDs to place the data blocks, $\mathrm{n}$ stands for id of data block, $\mathrm{k}$ represents the number of redundancy, $\mathrm{N}$ is the scope of hash function.

\section{Experiments and Results}

Chord as a typical DHT network, has the routing locating time $\mathrm{O}(\operatorname{logN})$. We use chord as DHT subnet of DHTPatch. In the experiments, the video code rate is $300 \mathrm{Kbps}$, data block size is $30 \mathrm{~kb}$, video playback rate is $300 / 30=10$. The underlying network topology is generated by GT-ITM package, and the experimental network topology conforms Transit-Stub model. In each simulation cycle, 5\% nodes join the network, and 5\% node leave the network. In all existing Gossip-based P2P streaming systems, CoolStreaming [7] system is the most representative because it's simple and practical. So in the simulation experiments, we also prepare a CoolStreaming simulation code, and compare the performance of DHTPatch to CoolStreaming in the same network environment .

\section{A. Message load}

In each scheduling cycle, its neighbor nodes exchange data availability messages, control overhead is defined as the ratio of data availability messages exchanged in communication message of system. Fig. 4 shows that the number of nodes does not affect the control overhead, as the same percentage of DHT nodes, the control overhead is maintained at a relatively low percentage. With the increase of the proportion of DHT nodes, the DHT network maintenance cost increases, the control overhead is also gradually increase, but it is limited to about $1.8 \%$ or less.

\section{B. Play continuity}

Improving the playback continuous degree in streaming media applications is one of the basic objectives. The playback continuity is defined as the probability that nodes can obtain sufficient data blocks, also can be understood as the ratio of nodes which can obtain sufficient data blocks in the network. Paper [7] defines continuity index as data segments arrived accounted for the proportion of the cache. Obviously, this does not guarantee smooth playback. Fig. 3 shows playback continuity track changes over time. At the first 5 seconds, CoolStreaming and DHTPatch trajectory is basically the same, because the DHT-Patch subnet data in DHT are not ready and limitedly contribute to the system; Playback continuity of DHT-Patch in 10 seconds reaches 0.8 degrees, while CoolStreaming reaches after 15 seconds, indicating that DHT-Patch system is on the average startup faster than CoolStreaming which is pure gossip multicasted system.

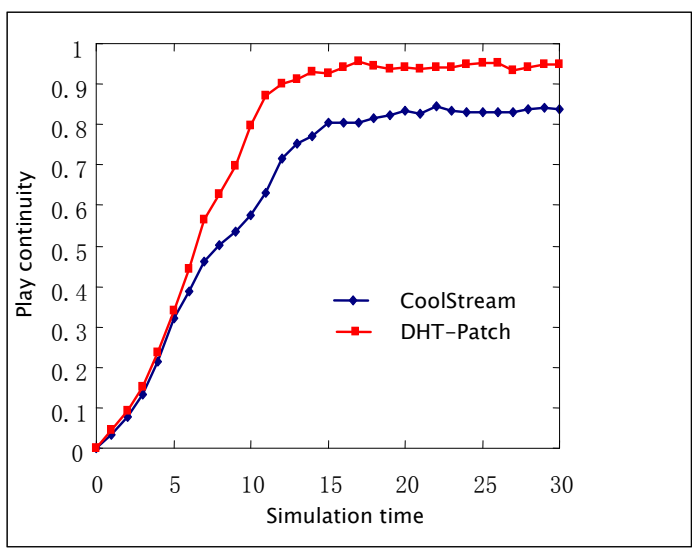

Fig. 3 The play continuity track with time

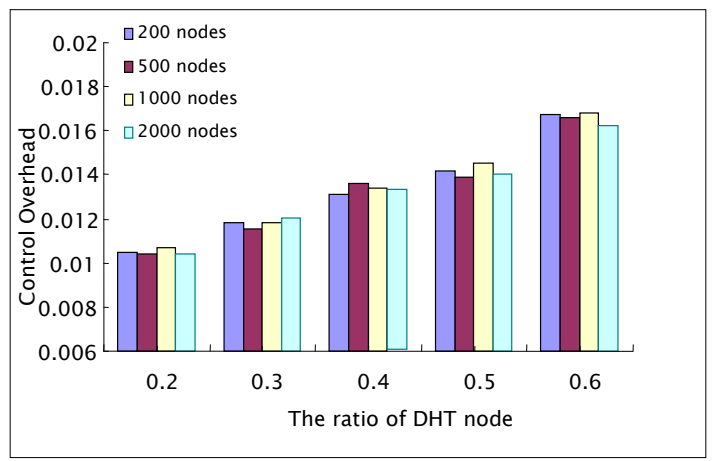

Fig. 4 Control overhead with different DHT networks 
Fig. 4 shows the evolutions of control overhead with different number of DHT network nodes. It gives us the hint to use the DHT network.

\section{Conclusion}

Gossip protocol-based P2P streaming systems get widely attention because of efficient work performance in a dynamic internet environment. However, existing systems can not ensure high play continuity caused by the randomness of data dissemination due to the Gossip protocol. Ignoring heterogeneity of network, system can not use resources effectively. In this paper, the high performance nodes constitute DHT subnet network for auxiliary to compensate for multicast defects of Gossip protocol, and we designed the DHTPatch streaming media system. Through simulation experiments, results proved its effectiveness. Next we hope to deploy the system in the actual network to discover more worthy of study in order to expand our thinking.

\section{Reference}

[1] Forecast C V N I. Cisco Visual Networking Index: Global Mobile data Traffic Forecast Update 2009-2014. Cisco Public Information, February, 2010, 9.

[2] Li Z, Cao J, Chen G. ContinuStreaming: Achieving high playback continuity of Gossip-based Peer-to-Peer streaming Parallel and Distributed Processing, 2008. IPDPS 2008. IEEE International Symposium on. IEEE, 2008: 1-12.

[3] Zhao L, Luo J G, Zhang M, et al. Gridmedia: A practical peer-to-peer based live video streaming system. Multimedia Signal Processing, 2005 IEEE 7th Workshop on. IEEE, 2005: 1-4.

[4] Yiu W P K, Jin X, Chan S H G. VMesh: Distributed segment storage for peer-to-peer interactive video streaming. Selected Areas in Communications, IEEE Journal on, 2007, 25(9): 1717-1731.

[5] Zhang X, Li Z, Zhang T, et al. RELookup: Providing Resilient and Efficient Lookup Service for P2P-VoD Streaming. Parallel and Distributed Systems (ICPADS), 2011 IEEE 17th International Conference on. IEEE, 2011: 747-752.

[6] Chen Zhuo,Feng Gang,Zhou Yang et al. SDHT: Efficient One- Hop DHT Lookup Framework for P2P Live Streaming. China Communications, 2012, 9(8): 88-104.

[7] Zhang X, Liu J, Li B, et al. DO-Net/CoolStreaming: A Data-Driven Overlay Network for Live Media Streaming. Proc. Conf. Computer Communications (INFOCOM). Miami, FL. 2005. 